\title{
Molecular targeted therapies in non-small cell lung cancer: where we are
}

In the last twenty years the landscape of treatment for advanced non-small cell lung cancer (NSCLC) has been totally revolutionized by the identification of somatic genetic alterations of DNA, specific for type of tumor, which are the molecular driving events for oncogenesis and, at the same time, very sensitive targets for individualized treatments.

In lung adenocarcinoma the oncogene addiction has been clearly demonstrated for a growing number of oncogenes, leading abnormalities at genetic, epigenetic or chromosomal level, which are critical events for the development of cancer (1).

Those abnormalities represent the rational basis for the so-called precision medicine in lung adenocarcinoma and their identification by the most sophisticated technology, such as next generation sequencing, allows the development of molecular targeted treatments which have deeply modified the natural history and the outcome of a significant number of lung adenocarcinomas.

The oncogene addiction in NSCLC has been shown for a number of oncogenes, such as EGFR mutations, EML4-ALK inversion, ROS1 rerrangements, RET translocations, cERBB2 mutations, BRAF mutations, c-MET mutations, NTRK rearrangements and KRAS mutations. The evidence of molecular alterations has led to the discovery of specific targetoriented drugs which have improved the outcome of the disease in terms of better response rate and prolonged progression free survival (PFS) and overall survival (OS).

The oncogene addiction in lung adenocarcinoma recognized a common mechanism of inducing and maintaining the cancer phenotype through the activation of unique, different and specific tyrosine kinases (TKs) which are the recognized target of molecular TK inhibitors (TKIs).

As well as the common mechanisms of inducing cell proliferation and evading apoptosis, the TK pathways show similar mechanisms of developing drug resistance by the appearance of new activating mutations or by the by-pass to new oncogenes as leading agents of cell proliferation (2).

In this issue of Translational Lung Cancer Research, the resistance of EGFR TKIs has been extensively described as a model, according to the largest experience of drug development and resistance insurgence in over 20 years of research and clinical trials in EGFR mutated patients (3).

Similarly the treatment evolution and the perspectives in ALK rearrangements are discussed in two different articles which describe the logical development of therapeutical strategies that in few years have faced the phenomena of drug resistance and the evolution of a number of new ALK TKIs with lower IC50 and different pharmacokinetics in respect to the first basic agent, crizotinib (4).

The strategies of development of new TKIs and the knowledge of resistance mechanisms to molecular targeted agents have been greatly facilitated by the implementation worldwide of the liquid biopsy which can allows the continuous detection in circulating tumor DNA or RNA of all the genetic, epigenetic of chromosomal abnormalities, as well as the introduction of a new concept of monitoring the natural history of the disease and the treatment response through an easier and reproducible biomarker. Liquid biopsy is still under continuous investigation and its role is expanding through the extensive application of new technologies which allow the detection either in tissue or in blood, of molecular alterations that need to be investigated in their influence and meaning on treatment decisions (5).

In oncogene addicted lung adenocarcinoma the incidence of brain metastasis seems to be higher because of an improved control of systemic disease, a better survival and, mainly in ALK rearranged patients, a natural propensity of central nervous system (CNS) disease localization: it's evaluated that the incidence of brain metastases in ALK rearranged patients is around $30 \%$ at first diagnosis and up to $60 \%$ during the history of these patients. Brain and CNS metastases are an important issue in oncogene addicted tumors, being often the unique failing site during first generation TKIs treatment, and a number of trials have addressed the utility of combining systemic TKIs with radiotherapy (6).

Recently third generation TKIs, either in EGFR mutated and ALK rearranged patients, have shown a better penetrance in cerebro-spinal fluid and their better activity on brain metastases are the major reason of superiority of osimertinib, alectinib and brigatinib $v s$. first generation TKIs in randomized clinical trials $(4,7)$. 
It's noteworthy that the third generation TKIs can also delay or prevent the appearance of brain metastases, sparing the necessity of radiotherapy and at the same time improving OS in this selected group of patients (6).

Oncogene addicted lung adenocarcinoma represent at the moment $25-30 \%$ of all adenocarcinoma with an increased number according to the better searching and diagnostic refinements. The prolonged survival, the continuous improvement in drugs availability and in the knowledge of the molecular characterization offer new opportunities to these patients population with a lower median age around 50-60 and represent one of the most important advances in modern medicine.

\section{Acknowledgments}

None.

\section{Footnote}

Conflicts of Interest: The authors have no conflicts of interest to declare.

Ethical Statement: The authors are accountable for all aspects of the work in ensuring that questions related to the accuracy or integrity of any part of the work are appropriately investigated and resolved.

\section{References}

1. Weinstein IB. Cancer. Addiction to oncogenes--the Achilles heal of cancer. Science 2002;297:63-4

2. Lin JJ, Shaw AT. Resisting resistance: targeted therapies in lung cancer. Trends Cancer 2016;2:350-64.

3. Paez JG, Sanne PA, Lee JC, et al. EGFR mutation in lung cancer: correlation with clinical response to gefitinib therapy. Science 2004;304:1497-500.

4. Peters S, Camidge DR, Shaw AT, et al. Alectinib versu crizotinib in untreated ALK positive no small-cell lung cancer. N Engl J Med 2017;377:829-38.

5. Alix-Panabières C, Pantel K. Clinical applications of circulating tumor cells and circulating tumor DNA as liquid biopsy. Cancer Discov 2016;6:479-91.

6. Rangachari D, Yamaguchi N, VanderLaan PA, et al. Brain metastases in patients with EGFR mutated or ALK-rearranged non small-cell lung cancer. Lung Cancer 2015;88:108-11.

7. Soria JC, Ohe Y, Vansteenkiste J, et al. Osimertinib in untreated EGFR-mutated advanced non small-cell lung cancer. N Engl J Med 2018;378:113-25.

Lucio Crinò, Angelo Delmonte Medical Oncology, Istituto Scientifico Romagnolo per lo Studio e la Cura dei Tumori (IRST) IRCCS, via Maroncelli, Meldola (FC), Italy.

(Email: lucio.crino@irst.emr.it) doi: $10.21037 /$ tlcr.2019.11.13

View this article at: http://dx.doi.org/10.21037/tlcr.2019.11.13

Cite this article as: Crinò L, Delmonte A. Molecular targeted therapies in non-small cell lung cancer: where we are. Transl Lung Cancer Res 2019;8(Suppl 3):S222-S223. doi: 10.21037/ tlcr.2019.11.13 